\title{
Profissionais de saúde mental: manifestação de stress e burnout ${ }^{1}$
}

\author{
Mental health professionals: manifestation \\ of stress and burnout
}

\author{
Ana Flávia de Oliveira SANTOS² \\ Carmen Lúcia CARDOSO
}

\begin{abstract}
Resumo
Profissionais de saúde mental, trabalhando em ambiente com elevada demanda emocional, encontram-se vulneráveis ao stress. Neste trabalho, objetivou-se avaliar manifestação de stress, burnout, autopercepção quanto ao stress e ao trabalho em 25 profissionais de serviços substitutivos em saúde mental. Aplicaram-se os instrumentos Inventário de Sintomas de Stress para Adultos de Lipp, Maslach Burnout Inventory e Roteiro Complementar. Dos participantes, 36\% apresentaram manifestação de stress, 44\% avaliaram-se como muito estressados e 60\% apresentaram alto esgotamento emocional. Para 60\%, o trabalho foi avaliado como muito estressante. As dimensões esgotamento emocional e despersonalização apresentaram correlações negativas com a variável idade. Conclui-se que há necessidade de intervenções de suporte ao trabalhador, principalmente aos mais jovens, direcionadas ao desenvolvimento de estratégias de enfrentamento às situações estressantes do trabalho.
\end{abstract}

Unitermos: Auto-imagem. Esgotamento profissional. Saúde mental.

\begin{abstract}
Mental health professionals, working in an environment with high emotional demands, find themselves vulnerable to stress. This study aimed to assess the manifestation of stress, burnout and self-perception regarding stress and work in 25 alternative mental health service professionals. The following instruments were employed:Lipp's Inventory for Stress Symptoms in Adults; Maslach Burnout Inventory and a Supplementary Script. The participants, presented manifestations of stress (36\%), rated themselves as being very stressed (44\%) and having high emotional burnout (60\%). For 60\%, work was evaluated as being very stressful. The dimensions of emotional burnout and depersonalization showed negative correlation to age variable. In conclusion, there is a need to provide workers with supportive interventions, especially to younger workers. In addition, interventions should be directed towards the development of coping strategies in stressful work situations.
\end{abstract}

Uniterms: Self-concept. Burnout, professional. Mental health.

A reforma psiquiátrica implantada no Brasil desde a década de 1980 tem produzido constantes e intensas transformações no modelo de assistência em saúde mental. A partir de diferenciações do modelo asilar, desenvolveu-se um dispositivo substitutivo de organização do cuidado em saúde mental caracterizado

\footnotetext{
$\boldsymbol{\nabla} \mathbf{\nabla} \boldsymbol{\nabla}$

1 Pesquisa financiada pela Fundação de Amparo à Pesquisa do Estado de São Paulo (Processo: 2005/04662-1).

2 Universidade de São Paulo, Faculdade de Filosofia, Ciências e Letras de Ribeirão Preto. Ribeirão Preto, SP, Brasil

3 Universidade de São Paulo, Faculdade de Filosofia, Ciências e Letras, Departamento de Psicologia e Educação, Programa de Pós-Graduação em Psicologia. Av. Bandeirantes, 3900, 14040-901, Ribeirão Preto, SP, Brasil. Correspondência para/Correspondence to: C.L. CARDOSO. E-mail: <carmen@ffclrp.usp.br>.
} 
pelo modo psicossocial de atenção. Estimulou-se, assim, a constituição de redes de atenção psicossocial que priorizam, entre outros aspectos, o trabalho em equipe multiprofissional, a reinserção social do usuário e a inclusão da família no tratamento (Costa-Rosa, 2000).

Esta reestruturação introduz novas exigências, relacionadas tanto à forma de se pensar e lidar com a doença mental, quanto à própria estruturação física e de organização da assistência aos usuários. Requer, pois, uma adaptação de todos os atores envolvidos no cuidado a uma lógica diferenciada de atendimento, demandando, principalmente por parte dos profissionais de saúde mental, o desenvolvimento de atitudes, habilidades e competências compatíveis com o modo psicossocial de atenção. Segundo Borges, Argolo e Baker (2006), mudanças no processo produtivo organizacional em diferentes setores têm implicado novas exigências de qualidade do serviço e o desenvolvimento de novas habilidades pelo trabalhador. Tais transformações incidem particularmente nos serviços de atenção à saúde, em que o lidar com o sofrimento, quer seja orgânico, emocional ou social, demanda uma carga suplementar de competências interpessoais.

Ramminger (2005), ao realizar uma pesquisa envolvendo profissionais de serviços substitutivos em saúde mental, descreve a percepção do profissional que, trabalhando neste contexto, é exigido quanto à necessidade de inovar em seu trabalho, devendo pensar sob uma lógica embasada em um discurso diferenciado, cuja prática, muitas vezes, não encontra os recursos necessários para uma atuação coerente com os princípios da reforma psiquiátrica, o que favorece o sentimento de sobrecarga nestes trabalhadores.

Uma vez que a categoria trabalho vem sendo considerada importante na saúde mental do indivíduo, atuando diretamente na relação saúde-doença (Silva \& Costa, 2008), tem-se observado uma preocupação com sua investigação junto a profissionais de saúde mental, especialmente considerando o contexto das transformações atuais neste campo. Estudos têm demonstrado que o trabalho na área, caracterizado pela atuação profissional em um ambiente com elevada demanda emocional, consiste em um fator significativo na manifestação de stress e da síndrome de burnout (Moore \& Cooper, 1996).

O stress foi caracterizado por Selye (1956) como 68 uma "Síndrome Geral de Adaptação" (SGA) decorrente de um evento que exige esforço do indivíduo em termos de adaptação. Este evento ambiental, denominado estressor, gera a quebra da homeostase interna do indivíduo, alterando a capacidade do organismo de manter sua constância. O referido autor dividiu o stress em três fases, a saber: a) alarme: reação de alerta, que prepara o indivíduo para a luta ou a fuga; b) resistência: tentativa de restabelecer o equilíbrio interno por meio de uma ação reparadora, gastando energia para esta adaptação; e c) exaustão: caracterizada pelo esgotamento físico e psicológico, momento em que as doenças são manifestadas, podendo ser fatais.

O estressor em si, no entanto, não determina toda a reação de stress. A percepção e a interpretação do indivíduo ante uma dada situação como excedendo seus recursos pessoais ou sobrecarregando-o são de demasiada importância para o desencadeamento do processo de stress. É, portanto, a partir da avaliação que o indivíduo faz de seu ambiente e de seus recursos pessoais que se acionam estratégias direcionadas a lidar com demandas internas e/ou externas desencadeadas por estressores (Lazarus \& Folkman, 1984).

Especificamente no que se relaciona ao stress no contexto ocupacional, destaca-se a questão do Burnout, um conceito que caracteriza uma síndrome psicológica produzida em resposta aos estressores interpessoais crônicos no trabalho. Tal conceito encontra-se associado à exaustão de energia decorrente de uma má adaptação a um trabalho estressante, prolongado e com elevada carga tensional, descrevendo uma condição de profissionais cujo trabalho requer elevado grau de contato interpessoal. O burnout manifesta-se através de três dimensões: a) esgotamento emocional: sentimento de estar sobrecarregado; b) despersonalização: surgimento de atitude de frieza e distanciamento direcionada aos colegas e ao trabalho; e c) reduzida realização pessoal: sentimentos de incompetência e falta de produtividade e realização no trabalho (Maslach, Schaufeli \& Leiter, 2001).

Por sua complexidade e especificidade, o trabalho em saúde mental é considerado de acentuada vulnerabilidade à sobrecarga emocional. Conforme assinalam Burrows e McGrath (2000), os estressores vivenciados por profissionais de saúde mental correspondem àqueles intrínsecos às profissões médicas. Dentre eles figura o lidar com pessoas que sofrem de transtornos 
mentais severos, considerado um dos fatores mais importantes para o desencadeamento do burnout.

Macedo (2005), ao investigar em uma população de profissionais de saúde mental as dificuldades encontradas por eles na execução de seu trabalho, encontrou os seguintes fatores: sobrecarga de trabalho, pressão em forma de cobrança excessiva, pouca valorização do trabalho, reduzido tempo para atendimentos, condições de trabalho inadequadas tanto materiais quanto técnicas, falta de treinamento e de capacitação de pessoal, falta de reconhecimento, desempenho de mais de uma função, baixa remuneração, falta de incentivo, falta de diálogo com as chefias, chefes imediatos sem capacitação técnica para a função e pouca preocupação com a qualidade dos serviços prestados. Na mesma perspectiva, Swoboda et al. (2006) encontraram que a ambiguidade de função, conflitos na equipe, doença do usuário e falta de tempo constituem aspectos do trabalho vivenciados como estressantes.

Silva e Costa (2008), considerando o paradigma do modelo psicossocial de atenção, constataram indicadores de sofrimento psíquico entre os profissionais, envolvendo dificuldades nas relações em equipe, com o usuário e com a organização. Quanto à aferição do burnout, Robinson, Clements e Land (2003) identificaram altos níveis de exaustão emocional em enfermeiros da área. Oliveira e Guerra (2004) não encontraram uma taxa de burnout acima da média em profissionais de saúde mental. Swoboda et al. (2005) encontraram baixo índice para as três dimensões em uma amostra de profissionais de saúde mental voltados à comunidade.

Os custos emocionais ao cuidador no contexto da assistência em saúde mental apontam para influências prejudiciais no desempenho, na qualidade do trabalho efetuado e na própria saúde do profissional, tornando imperativa uma investigação junto a esta população. Considerar a saúde e o bem-estar dos profissionais da saúde mental é importante porque problemas físicos e psicológicos podem conduzir a problemas somáticos variados, elevados níveis de ansiedade, depressão e problemas mentais, insônia, abuso deálcool e drogas, exaustão emocional, entre outros, como apontaram algumas pesquisas na área (Burrows \& McGrath, 2000; Hespanhol, 2005). Além disso, podem acarretar custos à organização, no tocante ao aumento de turnover e absenteísmo, levando à diminuição da eficiência e à reduzida satisfação profissional.

Com base nestas informações, este estudo objetivou avaliar a manifestação de stress e de burnout, a autopercepção quanto ao stress, bem como a percepção geral a respeito de o trabalho ser ou não fator estressante para profissionais de serviços substitutivos em saúde mental caracterizados pelo modo psicossocial de atenção.

\section{Método}

\section{Participantes}

Este estudo foi realizado com 25 trabalhadores de três serviços substitutivos, sendo dois ambulatoriais e um de internação parcial de saúde mental de uma cidade do interior do estado de São Paulo. Foram convidados a participar do estudo todos os profissionais que atendiam aos critérios de inclusão, a saber: ser trabaIhador de saúde mental contratado e estar empregado no serviço há, pelo menos, seis meses.

A amostra obtida correspondeu a 73,5\% da totalidade dos profissionais de serviços substitutivos da referida cidade. Constituiu-se por maioria do sexo feminino (76,0\%), idade predominante na faixa de 41 a 50 anos (36,0\%), estado civil casado (52,0\%). Dentre os participantes, 48,0\% possuíam o terceiro grau completo, seguido de 24,0\% que realizaram pós-graduação. Participaram assistentes sociais, enfermeiros, médicos psiquiatras, psicólogos e pessoal da área técnica.

O trabalho foi aprovado pelo Comitê de Ética em Pesquisa do Centro de Saúde Escola da Universidade de São Paulo (protocolo no 0167/CEP/CSE-USP), adotando-se os procedimentos básicos e éticos de respeito aos voluntários e à instituição, de acordo com a resolução no 196/96 sobre pesquisa envolvendo seres humanos (Brasil, 1996).

\section{Instrumentos} de dados:

Foram utilizados três instrumentos para a coleta

a) Inventário de Sintomas de Stress para Adultos de Lipp (ISSL): validado por Lipp (2000), permite identificar 
a presença de sintomas de stress, os tipos de sintomatologia - somática ou psicológica - e a fase de stress em que o indivíduo se encontra. O ISSL, composto por 53 itens divididos em três partes, avalia primeiramente os sintomas físicos e psicológicos das últimas 24 horas (15 itens); posteriormente, os sintomas manifestados na última semana (15 itens) e, por fim, os sintomas experimentados no último mês (23 itens). Refere bons indicadores de consistência interna (coeficiente alfa de cronbach 0,91).

b) Maslach Burnout Inventory (MBI): traduzido para o Brasil e autorizado para uso nesta pesquisa pela "Instrumento de Desenvolvimento Humano", distribuidora nacional do instrumento. Consta de 22 itens que avaliam índices de burnout subdivididos em três dimensões: Exaustão Emocional (EE), com nove itens, Despersonalização (DP), com cinco itens, e Realização Pessoal (RP), com oito itens. A pontuação total do questionário é o resultado da soma de todos os pontos das frequências respondidas nas dimensões do construto, podendo, cada uma delas, ser alta, média ou baixa. É utilizado para aferir o grau de incidência da síndrome de burnout nos participantes. Conforme Carlotto e Câmara (2007), o instrumento possui indicadores satisfatórios de consistência interna (coeficiente alfa de Cronbach variando de 0,65 a 0,94).

c) Roteiro Complementar (elaborado para esse estudo): composto por 15 itens abarcando os aspectos sociodemográficos dos trabalhadores, uma questão de avaliação da autopercepção do stress segundo uma escala do tipo Likert de 5 pontos, de intensidade variando de "nada estressado" a "muito estressado", bem como uma questão que propunha uma avaliação quanto à percepção de o trabalho em saúde mental ser ou não estressante, em função de uma escala de intensidade variando de "nada estressante" a "muito estressante".

\section{Procedimentos}

Os participantes responderam aos instrumentos na presença da primeira autora, que esteve disponível para eventuais esclarecimentos. Inicialmente, foi ressaltado o caráter voluntário da colaboração, esclarecendo-se as condições de sua participação através do Termo de Consentimento Livre e Esclarecido, que foi lido e 70 assinado pelos participantes. Os instrumentos foram aplicados segundo suas instruções particulares, na seguinte ordem - Roteiro Complementar, ISSL e MBI, sendo posteriormente cotados segundo suas proposições técnicas. Os dados foram codificados visando ao posterior tratamento estatístico. Para a definição do procedimento estatístico foi aplicado, inicialmente, o Teste Kolmogorov-Smirnov. Devido à não normalidade dos dados, foi efetuado o tratamento estatístico inferencial, mediante estatística não paramétrica, aplicando-se o Teste de Mann-Whitney e o Coeficiente de Correlação de Postos de Spearman, adotando-se valores de $p \leq 0,05$.

\section{Resultados}

Verificou-se, a partir dos resultados obtidos pelo ISSL, que 36,0\% dos participantes apresentaram indicadores de presença de manifestação de stress. Destes, 88,9\% encontravam-se na fase de resistência e 11,1\% na de exaustão. Quanto à sintomatologia apresentada, 66,7\% referiram predominantemente sintomas físicos.

Dos respondentes, 60,0\% apresentaram alto índice em exaustão emocional e 72,0\% alta realização pessoal. Quanto à despersonalização, entendida aqui como o surgimento de atitude de frieza e de distanciamento direcionada aos colegas e ao trabalho, 40,0\% apresentaram moderado índice, assim como 40,0\% demonstraram baixo nível nesta dimensão avaliada pelo $\mathrm{MBI}$.

Para 60,0\% dos profissionais, o trabalho foi avaliado como fator muito estressante. Em relação à percepção do próprio stress, 44,0\% avaliaram-se como muito estressados, segundo dados obtidos pelas escalas propostas no Roteiro Complementar.

Pela análise estatística inferencial das dimensões de burnout (MBI) e os indicadores de presença de stress (ISSL), verificou-se que os participantes com indicadores de presença de stress apresentaram valores superiores de esgotamento emocional, quando comparados àqueles com ausência de stress ( $U=31,00 ; z=-2,33 ; p=0,02$-Teste de Mann-Whitney). Não foram encontradas diferenças significativas para as demais dimensões de burnout.

Quanto às dimensões de burnout do MBI em comparação com a percepção do próprio stress (Roteiro 
Complementar), obtiveram-se valores superiores na dimensão esgotamento emocional entre participantes com percepção de si como muito estressados, quando comparados aos pouco estressados $(U=35,00 ; z=-2,04$; $p=0,04$ - Teste de Mann-Whitney). Para as demais dimensões do burnout, não foram encontradas diferenças significativas.

Em relação às variáveis sociodemográficas, foram obtidos valores significativamente superiores na dimensão da realização pessoal para o sexo feminino, quando comparado ao sexo masculino $(U=20,50$; $z=-2,33 ; p=0,01$ - Teste de Mann-Whitney). Para as demais dimensões do burnout, não houve diferenças significativas.

Foram encontradas correlações negativas entre a variável idade e a dimensão de esgotamento emocional ( $r=-0,47 ; p=0,01$ - Coeficiente de Correlação de Postos de Sperman) e correlação negativa entre a variável idade e a dimensão de despersonalização ( $r=-0,39 ; p=0,05$ - Coeficiente de Correlação de Postos de Sperman). Não foi encontrada correlação entre a dimensão realização pessoal e a variável idade.

\section{Discussão}

Os dados obtidos revelam que um número considerável dos profissionais de saúde mental estudados apresentou indicadores de manifestações de stress obtidos pelo ISSL. Este dado se mostra em concordância com estudos que apontam que os profissionais da área da saúde mental apresentam manifestações de stress (Carson \& Holloway, 2007; Prosser et al., 1999; Swoboda et al., 2005). Em estudos brasileiros, encontraram-se dados significativos em relação à presença de stress em técnicos e profissionais da área da saúde, verificando-se maioria com manifestações de stress (Carvalho \& Malagris, 2007; Malagris \& Fiorito, 2006).

Nesta pesquisa, dentre os profissionais que revelaram presença de stress, a maioria se situou na fase de resistência, fase esta em que o stress, já instalado, exige dispêndio de energia por parte do indivíduo na tentativa de restabelecer o equilíbrio interno por meio de ação reparadora, o que pode levar ao enfraquecimento físico do indivíduo e ao risco de desenvolver doenças. Vale ressaltar que, uma vez que a adaptabilidade do indi- víduo é finita (Selye, 1983), há a necessidade de que estes profissionais desenvolvam estratégias para melhor manejar estes estressores. Conseguindo controlá-los, seu organismo poderá retornar à estabilidade; no entanto, permanecendo sob pressão destes ou de novos estressores, poderão atingir a fase de exaustão física e psicológica, momento no qual estarão ainda mais vulneráveis ao adoecimento.

Os indicadores de stress apresentados pelos participantes são predominantemente de natureza física, manifestando-se por meio de sintomatologia como tensão muscular, taquicardia, dores de cabeça, desgaste físico constante, entre outros. Tais sintomas podem interferir negativamente na disposição do indivíduo, inclusive em suas atividades laborais voltadas ao cuidado do usuário.

Cabe ressaltar que um número maior de participantes se percebe como estressado quando comparado aos dados obtidos pelo ISSL. Estes resultados apontam para a relevância da avaliação destas duas dimensões do stress, uma vez que o processo de enfrentamento a uma situação estressante depende da percepção e análise do indivíduo quanto à sobrecarga causada pelo estressor.

Pode-se considerar que os profissionais de saúde mental, vivenciando stress com sintomatologia física, apresentam dificuldades em relação ao trabalho em saúde mental, tendo em vista que o seu trabalho exige contato direto com situações que levam à vulnerabilidade emocional. Para Moore e Cooper (1996), o ambiente de trabalho destes profissionais poderia levar à sua vulnerabilidade, à tensão psicológica e à exaustão emocional, devido às altas exigências emocionais que estes profissionais enfrentam. Carvalho e Malagris (2007) sugerem que o contato direto com o sofrimento do usuário no contexto da assistência em saúde torna o profissional suscetível às manifestações de stress.

Em relação à síndrome de burnout, pode-se dizer, de maneira geral, que os participantes da pesquisa vivenciam sentimentos de estarem sobrecarregados e esgotados (exaustão emocional), com surgimento de certa atitude de frieza e distanciamento em relação ao seu trabalho (despersonalização), apesar de apresentarem uma alta realização pessoal. A incidência de alta realização poderia refletir a satisfação por atuar no campo profissional de escolha que, ainda que apresente 
condições adversas, funciona como fator de realização pessoal no trabalho. O índice alto na dimensão realização pessoal, com o sentimento de gratificação pelo trabalho, pode contribuir até mesmo para atenuar a vivência do Burnout, contribuindo para a permanência do profissional em atividade (Moniz \& Araújo, 2006).

Os resultados de estudos que apontam para alto esgotamento emocional e stress, apesar da alta satisfação e realização pessoal, impulsionaram a investigação empreendida por Reid et al. (1999). Esta suposta contradição se deveria, no referido estudo, a ambivalências sentidas pelos profissionais de saúde mental, principalmente entre aqueles voltados à comunidade, quanto à avaliação de fatores do trabalho como sendo recompensadores e, ao mesmo tempo, estressores. Por exemplo, podem-se citar o senso de responsabilidade pelos atos e o bem-estar dos usuários que, apesar de satisfazerem o profissional, também o sobrecarregam.

Na relação entre percepção do próprio stress e Burnout, verificou-se que os participantes que se avaliaram como muito estressados também apresentaram maior sentimento de sobrecarga e esgotamento quanto ao trabalho. De acordo com Maslach et al. (2001), a exaustão emocional reflete a dimensão de stress da síndrome de burnout. Neste sentido, também foi encontrado que participantes com indicadores de presença de manifestação de stress e burnout apresentam-se intimamente atrelados.

Considerando que foram utilizados três instrumentos para avaliar a questão do stress, e uma vez que estes apontaram para a mesma direção, pode-se supor que o stress esteja relacionado ao trabalho em saúde mental. Corroborando esta hipótese, vale destacar que a maioria dos participantes avaliou o próprio trabalho como muito estressante.

Tais vivências de sobrecarga e stress podem estar relacionadas, entre outros aspectos, com as novas exigências e demandas que o trabalho em saúde mental atualmente requer. As instituições onde se realizou este estudo são fruto do processo de reforma da assistência psiquiátrica, nas quais os profissionais foram chamados a trabalhar sob uma lógica de assistência diferente da oferecida pela graduação quando de sua formação, pautada no modelo assistencial curativista-individual. Estes profissionais são exigidos quanto a novas habili72 dades para o desempenho do trabalho, tais como incluir o usuário como participante da equipe, incluir a família no processo de tratamento, trabalhar em sintonia em equipe multidisciplinar; devem, pois, ampliar o olhar para as questões psicossociais que permeiam o trabalho em saúde mental. Corroborando estes achados, vários autores discutem a questão da mudança de paradigma e os impactos na sobrecarga do trabalho (Silva \& Costa, 2008; Ramminger, 2005).

As relações entre os dados sociodemográficos e o Burnout apontaram que as mulheres apresentaram significativamente maior realização pessoal. A inserção feminina no contexto laboral pode atuar de maneira benéfica ao seu bem-estar pessoal, ainda que muitas vezes se submetam a uma dupla jornada de trabalho devido às responsabilidades de casa (Ludermir, 2000). Culturalmente, atividades de cuidado ao outro são responsabilidade feminina (Gil-Monte, 2002), o que também pode influenciar a satisfação das mulheres em relação ao trabalho caracterizado pela ajuda ao outro no campo da saúde mental.

Encontraram-se correlações negativas entre o fator esgotamento emocional e a variável idade, bem como entre o fator despersonalização e a variável idade, indicando que profissionais mais novos apresentam maior índice de esgotamento emocional e de despersonalização. O dado aponta para uma ligação entre idade e experiência no campo profissional, sugerindo que profissionais mais novos apresentam maior sentimento de sobrecarga pelo trabalho e atitude de distanciamento direcionada aos colegas e ao trabalho.

O estudo realizado por Rebouças, Legay e Abelha (2007) com trabalhadores em saúde mental encontrou dados semelhantes, constatando que os funcionários mais jovens, além de menos satisfeitos com o trabalho, também eram os que mais sofriam impactos do trabalho, especialmente no que diz respeito às repercussões emocionais. De Marco, Cítero, Moraes e Nogueira-Martins (2008) atribuem à experiência o papel de aumentar o controle e a segurança nas decisões e, assim, minorar o stress e a exaustão emocional.

Os dados referentes à sobrecarga de profissionais mais jovens assinalam a necessidade de acompanhamento do profissional, principalmente em início de carreira, através da proposição de atividades de supervisão e grupo de estudo, visando à instrumentalização 
técnica e compartilhamento de experiência, constituindo uma rede de apoio ao profissional. Ainda, a sistematização de dados quanto à inserção do jovem profissional no trabalho, o acompanhamento e a avaliação de seu trabalho, buscando identificar as dificuldades vivenciadas e os seus recursos potenciais, poderiam oferecer subsídios para a estruturação de programas de formação continuada que auxiliem na adaptação do profissional, refletindo, por conseguinte, em uma boa qualidade da assistência.

Assinala-se, ainda, a importância da psicoterapia objetivando o desenvolvimento de condições internas para o trabalho assistencial em saúde mental, particularmente tendo em vista a elevada demanda emocional envolvida neste cuidado. Pode-se sugerir também como alternativa no acompanhamento dos profissionais a implementação de tutoria, na qual se reconhece a capacidade de profissionais mais experientes em colaborar na formação dos mais novos, sendo estas iniciativas importantes para o cuidado e a promoção de saúde do próprio trabalhador de serviços de saúde mental.

Analisando-se alguns limites em relação aos aspectos metodológicos, aponta-se o número de participantes que compuseram a amostra deste estudo $(n=25)$. Tendo em vista que o estudo foi desenvolvido no contexto de três serviços substitutivos, a colaboração de $73,5 \%$ do total de profissionais indica uma amostra representativa das instituições existentes.

\section{Considerações Finais}

O estudo apontou indicadores de manifestações de stress, sobrecarga e esgotamento emocional, sugerindo que os trabalhadores apresentam dificuldades em relação ao trabalho em saúde mental, o que pode influenciar negativamente a qualidade da assistência prestada aos usuários. Apesar da percepção de stress advindo do trabalho, destaca-se a alta realização pessoal ligada ao trabalho entre os participantes da pesquisa, trazendo o indicativo de que a satisfação profissional se encontra para além das adversidades enfrentadas neste contexto. Profissionais mais jovens apresentaram significativamente maior esgotamento emocional e despersonalização, podendo apontar para o papel da experiência como um possível fator de proteção ao Burnout.
Tendo em vista a relevância destes achados, aponta-se a necessidade da criação de espaços para a construção de um novo tipo de trabalho que valorize a saúde do trabalhador, a cidadania, o respeito ao outro e à alteridade, instituindo-se programas voltados à saúde do trabalhador, visando ao desenvolvimento de recursos para lidar com o stress e com as dificuldades inerentes ao trabalho em saúde mental. Novos estudos poderiam se ocupar das relações existentes entre fatores que se encontram atrelados ao stress e ao burnout, destacando-se a relação existente entre indicadores de manifestações de stress, esgotamento emocional e realização e satisfação pessoal no trabalho, buscando desvelar seus significados no contexto da reforma psiquiátrica.

A identificação e caracterização das manifestações de stress e burnout no campo da saúde mental torna-se, pois, relevante, na medida em que favorece a reflexão acerca dos processos de trabalho e da qualidade de vida destes profissionais, necessária para o desenvolvimento de novas formas de pensar e fazer consoantes com a reforma psiquiátrica (Silva \& Costa, 2008). Estratégias de investigação sistemática junto a estes profissionais possibilitam uma reflexão acerca dos processos de trabalho, bem como a elaboração de propostas de intervenção que deem suporte para o profissional de saúde mental, sendo, pois, fundamentais para a proteção da saúde destes trabalhadores e, por conseguinte, da população atendida.

\section{Referências}

Borges, L. O., Argolo, J. C. T., \& Baker, M. C. S. (2006). Os valores organizacionais e a síndrome de burnout: dois momentos em uma maternidade pública. Psicologia: Reflexão e Crítica, 19 (1), 34-43.

Brasil. Ministério da Saúde. (1996). Resolução n 196/96 sobrepesquisa envolvendo seres humanos. Brasília: Conselho Nacional de Saúde.

Burrows, D., \& McGrath, C. (2000). Stress and mental health professionals. Stress Medicine, 16 (5), 269-270.

Carlotto, M. S., \& Câmara, S. G. (2007). Propriedades psicométricas do Maslach Burnout Inventory em uma amostra multifuncional. Estudos de Psicologia (Campinas), 24 (3), 325-32.

Carvalho, L., \& Malagris, L. E. N. (2007). Avaliação do nível de stress em profissionais de saúde. Estudo e Pesquisa em Psicologia, 7 (3), 210-221.

Carson, J., \& Holloway, F. (2007). Understanding and managing stress. In D. Bhugra \& O. Howes (Orgs.), 
Handbook for psychiatric trainees (pp.157-168). RCPsych Publications.

Costa-Rosa, A. (2000). O modo psicossocial: um paradigma das práticas substitutivas ao modo asilar. In P. Amarante (Org.), Ensaios: subjetividade, saúde mental e sociedade (pp.141-168). Rio de Janeiro: Ed. Fiocruz.

De Marco, P. F., Cítero, V. A., Moraes, E., \& Nogueira-Martins, L. A. (2008). O impacto do trabalho em saúde mental: transtornos psiquiátricos menores, qualidade de vida e satisfação profissional. Jornal Brasileiro de Psiquiatria, 57(3), 178-83.

Gil-Monte, P. R. (2002). Influencia del género sobre el proceso de desarrollo del síndrome de quemarse por el trabajo (Burnout) en profesionales de enfermería. Psicologia em Estudo, 7 (1), 3-10.

Hespanhol, A. (2005). Burnout e stress ocupacional. Revista Portuguesa de Psicossomática, 7 (1-2), 153-62.

Lazarus, R. S., \& Folkman, S. (1984). Stress, appraisal and coping. New York: Sringer Publishing Company.

Lipp, M. E. N. (2000). Inventário de Sintomas de Stress para adultos de Lipp (ISSL): manual. São Paulo: Casa do Psicólogo.

Ludermir, A. B. (2000). Inserção produtiva, gênero e saúde mental. Cadernos de Saúde Pública, 16 (3), 123-129.

Macedo, Z. D. (2005). Dificuldades que afetam os profissionais da saúde mental na rede pública da região oeste de Santa Catarina. Dissertação de mestrado não-publicada, Universidade do Oeste de Santa Catarina, Joaçaba.

Malagris, L. E. N., \& Fiorito, A. C. C. (2006). Avaliação do nível de stress de técnicos da área da saúde. Estudos de Psicologia (Campinas), 23 (4), 391-398.

Maslach, C., Schaufeli, W. B., \& Leiter, M. P. (2001). Job burnout. Annual Review Psychology, 52 (1), 397-422.

Moniz, A. L. F., \& Araújo, T. C. C. F. (2006). Trabalho voluntário em saúde: auto-percepção, estresse e burnout. Interação em Psicologia, 10 (2), 235-243.

Moore, K. A., \& Cooper, C. L. (1996). Stress in mental health professionals: a theoretical overview. International Journal of Social Psychiatry, 42 (2), 82-89.

Oliveira, M., \& Guerra, M. P. (2004). Burnout nos profissionais de saúde mental: expectativas, auto-actualização e outras variáveis associadas. Revista Saúde Mental, 4 (1), 15-25.
Prosser, D., Johnson, S., Kuipers, E., Dunn, G., Szmukler, G., Reid, Y., et al., (1999). Mental health, "burnout", and job satisfaction in a longitudinal study of mental health staff. Social Psychiatry Epidemiology, 34 (6), 295-300.

Ramminger, T. (2005). Trabalhadores de saúde mental: reforma psiquiátrica, saúde do trabalhador e modos de subjetivação nos serviços de saúde mental. Dissertação de mestrado não-publicada, Universidade Federal do Rio Grande do Sul, Porto Alegre.

Rebouças, D., Legay, L. F., \& Abelha, L. (2007). Satisfação com o trabalho e impacto causado nos profissionais de serviço de saúde mental. Revista Saúde Pública , 41 (2), 244-250.

Reid, Y., Johnson, S., Morant, N., Kuipers, E., Szmukle, G., Thornicroft, G., et al., (1999). Explanations for stress and satisfaction in mental health professionals: a qualitative study. Social Psychiatry and Psychiatric Epidemiology, 34 (6), 301-308.

Robinson, J. R., Clements, K., \& Land, C. (2003). Workplace stress among psychiatric nurses. Prevalence, distribution, correlates, predictors. Journal Psychosocial Nursing Mental Health Services, 41 (4), 32-41.

Selye, H. (1956). The stress of life. New York: Longmans.

Selye, H. (1983). The Stress concept: past, present and future. In C. L. Cooper (Ed.), Stress research: issues for the eighties (pp.1-20). New York: John Wiley \& Sons.

Silva, E. A., \& Costa, I. I. (2008). Saúde mental dos trabalhadores em saúde mental: estudo exploratório com os profissionais dos Centros de Atenção Psicossocial de Goiânia/GO. Psicologia em Revista, 14 (1), 83-106.

Swoboda, H, Sibitz, I., Fruhwald, S., Klug, G., Bauer, B., \& Priebe, S. (2005). Job satisfaction and burnout in professionals in austrian mental health services. Psychiatrische Praxis, 32 (8), 386-392.

Swoboda, H, Sibitz, I., Fruhwald, S., Klug, G., Bauer, B., Priebe, S. (2006). How do community-based mental health staff in Austria perceive their job? A qualitative study. Psychiatrische Praxis, 32 (8), 386-392.

Recebido em: 27/1/2009

Versão final reapresentada em: 18/6/2009

Aprovado em: 23/9/2009 